\title{
INTERPRETING BEHAVIORAL, BIOMEDICAL, AND PSYCHOLOGICAL RELATIONSHIPS IN CHRONIC DISEASE FROM $2 \times 2$ TABLES USING CORRELATION
}

\author{
Fredric M. Wolf ${ }^{1}$ and Richard G. CoRnell ${ }^{2}$ \\ 'Department of Postgraduate Medicine and Health Professions Education, Medical School, \\ ${ }^{2}$ Department of Biostatistics, School of Public Health, and \\ ${ }^{1,2}$ Diabetes Research and Training Center, University of Michigan, \\ Ann Arbor, MI 48109, U.S.A.
}

(Received in revised form 2 January 1986)

\begin{abstract}
Correlations of 0.3 and 0.4 in psychological studies in chronic illness (e.g. diabetes) have been criticized as having little clinical importance. The present article suggests that correlation coefficients be interpreted as binomial effect size displays (BESD) as a means of gleaning the clinical importance of a given correlation coefficient. The BESD is easily understood, computed, and applied to research in chronic disease. An illustration is provided in which a $r$ of 0.2 is shown to correspond to a BESD in which the success rate is improved from 40 to $60 \%$. Using BESD to interpret the meaningfulness of relationships, correlations as low as 0.2 may have clinical importance.
\end{abstract}

CORRELATION coefficients of 0.3 in psychological, biomedical, and behavioral studies of chronic illness have been criticized as having no clinical relevance [1,2]. In an editorial in Diabetes Care, Skyler noted the importance of hehavioral, psychological, and social issues in the understanding, management, and impact of diabetes. He went on to caution that, "With a large enough sample, even correlation coefficients of 0.3 or 0.4 may reach statistical significance. These are not likely to be important correlations. Mathematics alone cannot be used to determine this." While it is true that very small correlations can reach statistical significance with a sufficiently large sample, the statement that correlations of 0.3 or 0.4 are not likely to be clinically relevant may be unfounded. Statements of this type are not uncommon and Skyler by no means is alone in advocating this view. This interpretation may stem, in part, from the prevalent belief that descriptive, exploratory, and correlational studies are by definition less rigorous and worthy than experimental, clinical trial type designs.

What is needed is greater emphasis on the magnitude of the effect observed when the sample size is sufficiently large so that statistical significance is attained and the correlation is estimated precisely (e.g. with a narrow confidence interval). For example, Cohen [3] provides guidelines for interpreting the effect size of correlation coefficients as small $(r=0.1)$ medium $(r=0.3)$ or large $(r=0.5)$ in the absence of well articulated criteria based upon the research literature concerning the question or hypothesis of interest. Peduzzi et

We thank the anonymous referees for their helpful comments and suggestions.

This work was supported in part by Grant No. NIH 5 P60 AM20572 from the National Institute of Arthritis, Diabetes, and Digestive and Kidney Diseases.

Address reprint requests and correspondence to Fredric M. Wolf, Department of Postgraduate Medicine and Health Professions Education, University of Michigan, G1208 Towsley Center (Box 57), Ann Arbor, MI 48109, U.S.A. 
al. [4] noted that the importance of a small correlation coefficient between two dichotomous variables may be more than is readily apparent because the range of maximum possible values may be restricted by the marginal totals. They suggested interpreting a correlation coefficient relative to the maximum possible correlation imposed by restrictions of the marginals. Another possible solution for approaching the magnitude of effect of a correlation coefficient that we propose in the present article is to interpret it as the estimated difference in success probabilities between treatment and control group conditions in a binomial effect size display (BESD) for $2 \times 2$ tables. The purpose of this article is to present this method for interpreting correlations and to provide an illustration of its usefulness in research and practice related to chronic disease.

This procedure was discussed by Rosenthal and Rubin [5] and is based upon the mathematical transformation of a correlation coefficient $(r)$ and chi square $\left(\chi^{2}\right)$ for $2 \times 2$ tables. The BESD is the estimated difference in success probabilities between treatment and control group conditions. Practically speaking, this transformation is simple to calculate, easily understood, and enables one to interpret $r$ in terms of the effect on success rates such as survival, improvement, or cure rates attributable to certain treatment procedures.

For example, suppose an $r$ of 0.2 is calculated for a $2 \times 2$ table comparing a treatment and control with respect to improvement of a chronic condition, as in Table 1. Suppose the sample size is large enough so that the null hypothesis that the two conditions is zero is rejected, as it would be for Table 1 with $N=200$. Despite the statistical significance of $r$, some individuals would dismiss the relationship between treatment and improvement as unimportant because $r^{2}$ is only 0.04 , which means that only $4 \%$ of the variance, a relatively small amount, is accounted for. However, the $r$ of 0.2 corresponds to increasing a success rate from 40 to $60 \%$ by means of an experimental intervention. This would mean, for example, that the improvement rate under the treatment condition is $60 \%$, but is only $40 \%$ under the control conditon as shown in Table 1. This is hardly a trivial, unimportant relationship $(r)$ once the corresponding BESD is calculated and examined. The BESD experimental success rate is computed as $0.5+r / 2$, whereas the BESD control success rate is computed as $0.5-r / 2$. Rosenthal and Rubin show that approximating the BESD by $r$ often yields a fairly realistic representation of the treatment effect size for continuously distributed variables and not just dichotomously distributed variables. The equality of $r$ and the improvement rate holds whenever the marginal distributions and entries of the $2 \times 2$ table are symmetric and equal (as in Table 1), and is fairly robust with respect to a fairly broad range of deviations from this symmetry ranging from 0.2 to 0.8 as illustrated in the Appendix. It is important to remember that behavior is complexly determined and that no relationships between two variables are unaffected by other, often unmeasured, factors. However, if there is indeed a true relationship of 0.2 between treatment conditions and success rates, then this relationship should translate into approximately a $20 \%$ change in success rates, all other things being equal. The formula for this transformation, an illustration, and proof of this relationship are provided in the Appendix.

Table 2 provides a summary of the corresponding BESD values and changes in success rates associated with various values of $r$. Certainly correlations of $0.2,0.3$ and 0.4 are clinically important and relevant if based on statistically significant results obtained from large numbers of representative subjects. Thus a reported correlation of 0.35 between glycosolated hemoglobin levels $\left(\mathrm{HbA}_{1}\right)$ and the extent to which diabetic adolescents follow their prescribed diet [7] translates into a success rate improvement in metabolic control from 33 to $67 \%$ with dietary adherence. Similarly, a reported correlation of 0.33 between

Table 1. Illustration of the binomial efFect sIze DISPLAY FOR A CORRELATION OF 0.2 BETWEEN CONDITION AND OUTCOME

\begin{tabular}{lccc}
\hline & \multicolumn{2}{c}{ Treatment outcome } & \\
\cline { 2 - 3 } Condition & Improvement & No improvement & Total \\
\hline Treatment & 60 & 40 & 100 \\
Control & 40 & 60 & 100 \\
Total & 100 & 100 & 200 \\
\hline
\end{tabular}


TABLE 2. BINOMIAL EFFECT SIZE DISPLAYS FOR VARIOUS CORRELATION COEFTICIENTS

\begin{tabular}{ccccc}
\hline & $\begin{array}{c}\text { Amount of } \\
\text { shared variance }\end{array}$ & & \multicolumn{2}{c}{$\begin{array}{c}\text { Success rate } \\
\text { increased }\end{array}$} \\
\cline { 2 - 3 } \cline { 5 - 5 }$r$ & $r^{2}$ & & From & To \\
\hline 0.10 & 0.01 & & 0.45 & 0.55 \\
0.20 & 0.04 & & 0.40 & 0.60 \\
0.30 & 0.09 & & 0.35 & 0.65 \\
0.40 & 0.16 & & 0.30 & 0.70 \\
0.50 & 0.25 & & 0.25 & 0.75 \\
0.60 & 0.36 & & 0.20 & 0.80 \\
0.70 & 0.49 & & 0.15 & 0.85 \\
0.80 & 0.64 & & 0.10 & 0.90 \\
0.90 & 0.81 & & 0.05 & 0.95 \\
\hline Success & rates $=0.5 \pm(r / 2)$ & Source: & Adapted
\end{tabular}

from Rosenthal and Rubin (1982).

$\mathrm{HbA}_{1}$ levels and diabetes-specific social and psychological problems [8] could be viewed as a reduction in the rates of psychological problems from 66 to $34 \%$ with normal metabolic control $\left(\mathrm{HbA}_{1}\right)$. It is apparent from Table 2 that the improvement in success rate is equivalent to the magnitude of $r$. That is, a correlation of 0.4 translates into a $40 \%$ improvement $(70-30 \%)$.

In summary, it is hoped that researchers, clinicians, and educators will begin to interpret correlation coefficients with their corresponding binomial effect size display changes in success rates. This measure is easily obtained and understood and hopefully will lead to a re-evaluation of the role of correlations in research in chronic disease. In practice, it would be helpful for researchers to present the estimated BESD equal to the difference in success proportions for the treatment $\left(p_{\mathrm{t}}\right)$ and control $\left(p_{\mathrm{c}}\right)$ groups obtained from $r$ or $\chi^{2}$ (1). These BESD success proportion rates also might be helpful in interpreting other test statistics, such as $t$-tests or $F$-ratios. Cohen [6], Friedman [9], and Rosenthal [10] provide formulae for computing the $r$ associated with a number of other test statistics. Once the $r$ associated with one of these other test statistics isobtained, the BESD success proportion rates can be calculated in a straightforward fashion as described. These formulae for calculating $r$ from $t$-test and $F$-ratio analysis of variance results also are provided in the appendix.

\section{REFERENCES}

1. Rimland B: Death knell for psychotherapy? Am Psychol 34: 192, 1979

2. Skyler JS: Psychological issues in diabetes (Editorial). Diabetes Care 4: 656-657, 1981

3. Cohen J: Statistical Power Analysis for the Behaviorial Sciences, revised edn. New York: Academic Press, 1977

4. Peduzzi PN, Detre KM, Chan YK: Upper and lower bounds for correlations in $2 \times 2$ tables-revisited. J Chron Dis 36: 491-496, 1983

5. Rosenthal R, Rubin DB: A simple, general purpose display of magnitude of experimental effect. J Educ Psychol 74: 166-169, 1982

6. Cohen J: Some statistical issues in psychological research. In Handbook of Clinical Psychology, Wolman BB (Ed.). New York: McGraw-Hill, 1965

7. Schafer LC, Glasgow RE, McCaul KD, Dreher M: Adherence to IDDM regimens: Relationship to psychosocial variables and metabolic control. Diabetes Care 6: 493-498, 1983

8. Wolf FM: Psychosocial factors in diabetes: A cognitive psychological approach. (Abstract). Diabetes 33 (Suppl 1): 28A, 1984

9. Friedman H: Magnitude of experimental effect and a table for its rapid estimation. Psychol Bull 70: 245-25I, 1968

10. Rosenthal R: Assessing the statistical and social importance of the effects of psychotherapy. J Consult Clin Psychol 51: 4-13, 1983

\section{APPENDIX}

The following illustration shows how $r$ is obtained from Table 1 using the equation provided by Cohen [6] where $A$ and $B$ are the frequencies in the first row of the table and $C$ and $D$ in the second row, and where $N=A+B+C+D$. 


$$
\begin{aligned}
r & =\sqrt{\frac{\chi^{2}(1)}{N}} \\
\chi^{2}(1) & =\frac{(A D-B C)^{2} N}{(A+B)(C+D)(A+C)(B+D)} \\
\chi^{2}(1) & =\frac{\left[(60)^{2}-(40)^{2}\right]^{2}(200)}{(100)(100)(100)(100)} \\
\chi^{2}(1) & =8 \\
r & =\sqrt{\frac{8}{200}}=0.2 .
\end{aligned}
$$

The BESD is the estimated difference in success proportions on treatment and control, $p_{\mathrm{t}}-p_{\mathrm{c}}$, where $p_{\mathrm{t}}>p_{\mathrm{c}}$ is reflected in a positive correlation coefficient $r$. Now $r=\mathrm{BESD} / f$ so that $r$ can be interpreted as BESD when $f$ is close to 1 . Let $k N$ denote the number of patients on treatment and $(1-k) N$ the number on control. Then

$$
f=[\bar{p}(1-\bar{p}) / k(1-k)]^{1 / 2} ; \quad \bar{p}=k p_{\mathrm{t}}+(1-k) p_{\mathrm{c}} .
$$

The factor $f$ is equal to one or close to one for $\bar{p}$ and $k$ in interals around 0.5 as shown in the following table:

$\begin{array}{ccc}k & \bar{p} & f \\ 0.5 & 0.5 & 1.00 \\ & 0.4 \text { or } 0.6 & 0.98 \\ 0.4 \text { or } 0.6 & 0.2 \text { or } 0.8 & 0.89 \\ & 0.5 & 1.02 \\ & 0.4 \text { or } 0.6 & 1.00 \\ 0.2 \text { or } 0.8 & 0.2 \text { or } 0.8 & 0.81 \\ & 0.5 & 1.25 \\ & 0.4 \text { or } 0.6 & 1.22 \\ & 0.2 \text { or } 0.8 & 1.00\end{array}$

From this table it can be seen that the interpretation of $r$ as BESD $(f=1)$ holds at least approximately for $\bar{p}$ in a middle range about 0.5 , as stated by Rosenthal and Rubin [5] provided $k$ is also in such a range. Moreover, $r$ is exactly equal to BESD when $k=1-p$ or $k=p$, including the special case when both $p$ and $k$ are 0.5. This case was presented by Rosenthal and Rubin [5] and is illustrated in Table 1 in this paper. The following formula may be used for computing the $r$ associated with $t$-test results.

$$
r=\sqrt{\frac{t^{2}}{t^{2}+d f}}
$$

Similarly, $r$ may be computed from $F$-ratios obtained from analysis of variance results comparing two group means (e.g. treatment and control), where the numerator degrees of freedom are equal to one.

$$
r=\sqrt{\frac{F}{F+d f(\text { error })}} .
$$

Once $r$ is obtained from its corresponding $t$-test or $F$-ratio, it is taken as an approximation to the BESD as described above. 